\title{
The Structure of Hopf Algebras Acting on Dihedral Extensions
}

\author{
Alan Koch, Timothy Kohl, Paul J. Truman, and Robert Underwood
}

\section{Introduction}

Galois theory for purely inseparable field extensions was first considered by Jacobson [15]. More broadly, Chase and Sweedler defined the notion of a Hopf algebra acting on a purely inseparable extension of fields to obtain a weak analogue to the Fundamental Theorem of Galois theory [6]. The construction of these "HopfGalois" structures however applies not only to purely inseparable field extensions but also to separable field extensions, as well as extensions of commutative rings (which will not be considered here).

In the groundbreaking paper [13], Greither and Pareigis obtain a classification of Hopf-Galois structures on separable field extensions $L / K$. The most remarkable aspect of their classification is that it is entirely group-theoretic, depending on $\operatorname{Gal}(E / K)$ and $\operatorname{Gal}(E / L)$, where $E$ is the normal closure of $L / K$ : the structure of $L / K$ is irrelevant (aside from these Galois groups). Much of the work to date has focused on counting the number of Hopf-Galois extensions, either directly (see, e.g., [4],[5],[8],[12],[18],[19],[20],[21]) or through results which facilitate computations (e.g., [2]).

In the three decades since the publication of [13], what has been lacking is a thorough investigation into the structure of the Hopf algebras which produce these Hopf-Galois structures. It is unclear how much the structure of the Hopf algebras also depends on group theory. To this end, we introduce some questions for study, including:

1. Can a single $K$-Hopf algebra determine more than one Hopf-Galois structure on $L / K$ ?

\footnotetext{
Alan Koch

Department of Mathematics, Agnes Scott College, 141 E. College Ave., Decatur, GA 30030 USA, e-mail: akoch@agnesscott.edu

Timothy Kohl

Department of Mathematics and Statistics, Boston University, 111 Cummington Mall, Boston, MA 02215 USA, e-mail: tkohl@math.bu.edu

Paul J. Truman

School of Computing and Mathematics, Keele University, Staffordshire, ST5 5BG, UK, e-mail: P.J.Truman@Keele.ac.uk

Robert Underwood

Department of Mathematics and Computer Science, Auburn University at Montgomery, Montgomery, AL, 36124 USA, e-mail: runderwo@aum.edu
} 
2. Can two non-isomorphic $K$-Hopf algebras, each of which giving a Hopf-Galois structure on $L / K$, become isomorphic upon base change to some intermediate field $K \subset F \subset L$ ?

3. Can a single $K$-algebra be endowed with multiple coalgebra structures, resulting in multiple (nonisomorphic) Hopf algebras, giving different Hopf-Galois structures on $L / K$ ?

4. Can two non-isomorphic $K$-algebras, each of which giving a Hopf-Galois structure on $L / K$ (after being endowed a coalgebra structure), become isomorphic as algebras upon base change to some intermediate field $K \subset F \subset L$ ?

It is not known whether the answers to these questions depend on knowledge of the fields, aside from their automorphism groups. Some specific cases-most notably the cases where $\mathrm{Gal}(L / K)$ is cyclic or elementary abelian-have been investigated by the authors in [17]. We note that L. Childs [10, Theorem 5] has shown that abelian fixed-point free endomorphisms of $\operatorname{Gal}(L / K)$ determine Hopf Galois structures on $L / K$ whose Hopf algebras are isomorphic to $H_{\lambda}$ (see Example 3 for the definition of $H_{\lambda}$ ). Childs applies his result to the cases where $\operatorname{Gal}(L / K)$ is the symmetric group $S_{n}, n \geq 5$, and the dihedral group of order $4 n, n \geq 2$. Thus in these cases, Childs has obtained an affirmative answer to Question (1). In this paper, we will focus on the case where $L / K$ is Galois with $\operatorname{Gal}(L / K)=D_{p}$, the dihedral group of order $2 p$ for some prime $p \geq 3$. In this instance, the questions above do in fact have satisfying group theoretic answers.

We start by reviewing Hopf-Galois theory and the Greither-Pareigis theory that yields the classification of Hopf-Galois structures in the separable case. We then apply Greither-Pareigis theory to describe the HopfGalois structures in the case that $L / K$ is Galois with group $D_{p}$; there are $p+2$ such structures, and while this was known previously (see [4]) we provide a simpler description. We will show that there are three Hopf algebra isomorphism classes, and that base changing to a proper intermediate field still results in three distinct Hopf algebra classes. On the other hand, there are two $K$-algebra isomorphism classes, and base changing to any intermediate field (even $L$ itself) does not change these isomorphism classes. We then find explicit bases for each of our Hopf algebras, and specializing to the case $p=3$, we give an even more detailed description of the algebra structure.

In contrast, we point out that the Hopf-Galois theory in the purely inseparable case differs greatly to what is presented here. For example, if $L=K(x), x^{p} \in K, x \notin K$, is a purely inseparable extension ( $p$ prime), then $H=K[t] /\left(t^{p}\right), t$ primitive, can act in an infinite number of ways (see, e.g., [16]), allowing for an infinite number of Hopf-Galois structures.

\section{Hopf Galois Theory}

In this section we recall the notion of a Hopf algebra, a Hopf-Galois extension, and the Greither-Pareigis classification.

A bialgebra over a field $K$ is a $K$-algebra $B$ together with $K$-algebra maps $\Delta: B \rightarrow B \otimes_{K} B$ (comultiplication) and $\varepsilon: B \rightarrow K$ (counit) which satisfy the conditions

$$
\begin{gathered}
(I \otimes \Delta) \Delta=(\Delta \otimes I) \Delta, \\
m u l t(I \otimes \varepsilon) \Delta=I=\operatorname{mult}(\varepsilon \otimes I) \Delta,
\end{gathered}
$$

where mult $: B \otimes_{K} B \rightarrow B$ is the multiplication map of $B$ and $I$ is the identity map on $B$. A Hopf algebra over $K$ is a $K$-bialgebra $H$ with a $K$-linear map $\sigma: H \rightarrow H$ which satisfies

$$
\operatorname{mult}(I \otimes \sigma) \Delta(h)=\varepsilon(h) 1_{H}=\operatorname{mult}(\sigma \otimes I) \Delta(h),
$$


for all $h \in H$. A $K$-Hopf algebra $H$ is cocommutative if $\Delta=\tau \circ \Delta$, where $\tau: H \otimes_{K} H \rightarrow H \otimes_{K} H, a \otimes b \mapsto b \otimes a$ is the twist map.

Let $L$ be a finite extension of $K$ and let $\mathrm{m}: L \otimes_{K} L \rightarrow L$ denote multiplication in $L$. Let $H$ be a finite dimensional, cocommutative $K$-Hopf algebra and suppose there is a $K$-linear action of $H$ on $L$ which satisfies

$$
\begin{aligned}
h \cdot(x y) & =(\mathrm{m} \circ \Delta)(h)(x \otimes y) \\
h \cdot 1 & =\varepsilon(h) 1
\end{aligned}
$$

for all $h \in H, x, y \in L$, and that the $K$-linear map $j: L \otimes_{K} H \rightarrow \operatorname{End}_{K}(L), j(x \otimes h)(y)=x(h \cdot y)$ is an isomorphism of vector spaces over $K$. Then we say $H$ provides a Hopf-Galois structure on $L / K$.

Example 1. Suppose $L / K$ is Galois with Galois group $G$. Let $H=K[G]$ be the group algebra, which is a Hopf algebra via $\Delta(g)=g \otimes g, \varepsilon(g)=1, \sigma(g)=g^{-1}$, for all $g \in G$. The action

$$
\left(\sum r_{g} g\right) \cdot x=\sum r_{g}(g(x))
$$

provides the "usual" Hopf-Galois structure on $L / K$ which we call the classical Hopf-Galois structure.

In general, the process of finding a Hopf algebra and constructing an action may seem daunting, but in the separable case Greither and Pareigis [13] have provided a complete classification of such structures. Let $L / K$ be separable with normal closure $E$. Let $G=\operatorname{Gal}(E / K), G^{\prime}=G a l(E / L)$, and $X=G / G^{\prime}$. Denote by $\operatorname{Perm}(X)$ the group of permutations of $X$. A subgroup $N \leq \operatorname{Perm}(X)$ is regular if $|N|=|X|$ and $\eta\left[x G^{\prime}\right] \neq x G^{\prime}$ for all $\eta \neq 1_{N}, x G^{\prime} \in X$. Let $\lambda: G \rightarrow \operatorname{Perm}(X), \lambda(g)\left(x G^{\prime}\right)=g x G^{\prime}$, denote the left translation map. A subgroup $N \leq \operatorname{Perm}(X)$ is normalized by $\lambda(G) \leq \operatorname{Perm}(X)$ if $\lambda(G)$ is contained in the normalizer of $N$ in $\operatorname{Perm}(X)$.

Theorem 1 (Greither-Pareigis). Let $L / K$ be a finite separable extension. There is a one-to-one correspondence between Hopf Galois structures on $L / K$ and regular subgroups of $\operatorname{Perm}(X)$ that are normalized by $\lambda(G)$.

One direction of this correspondence works by Galois descent: Let $N$ be a regular subgroup normalized by $\lambda(G)$. Then $G$ acts on the group algebra $E[N]$ through the Galois action on $E$ and conjugation by $\lambda(G)$ on $N$, i.e.,

$$
g(x \eta)=g(x)\left(\lambda(g) \eta \lambda\left(g^{-1}\right)\right), g \in G, x \in E, \eta \in N .
$$

For simplicity, we will denote the conjugation action of $\lambda(g) \in \lambda(G)$ on $\eta \in N$ by ${ }^{g} \eta$. We then define

$$
H=(E[N])^{G}=\{x \in E[N]: g(x)=x, \forall g \in G\} .
$$

The action of $H$ on $L / K$ is thus

$$
\left(\sum_{\eta \in N} r_{\eta} \eta\right) \cdot x=\sum_{\eta \in N} r_{\eta} \eta^{-1}\left[1_{G}\right](x)
$$

see [9, Proposition 1].

The fixed ring $H$ is an $n$-dimensional $K$-Hopf algebra, $n=[L: K]$, and $L / K$ has a Hopf Galois structure via $H$ [13, p. 248, proof of 3.1 (b) $\Longrightarrow$ (a)], [7, Theorem 6.8, pp. 52-54]. By [13, p. 249, proof of 3.1, (a) $\Longrightarrow$ (b)],

$$
E \otimes_{K} H \cong E \otimes_{K} K[N] \cong E[N],
$$


as $E$-Hopf algebras, that is, $H$ is an $E$-form of $K[N]$.

Theorem 1 can be applied to the case where $L / K$ is Galois with group $G$ (thus, $E=L, G^{\prime}=1_{G}, G / G^{\prime}=G$ ). In this case the Hopf Galois structures on $L / K$ correspond to regular subgroups of $\operatorname{Perm}(G)$ normalized by $\lambda(G)$, where $\lambda: G \rightarrow \operatorname{Perm}(G), \lambda(g)(h)=g h$, is the left regular representation.

Example 2. Suppose $L / K$ is a Galois extension, $G=\operatorname{Gal}(L / K)$. Let $\rho: G \rightarrow \operatorname{Perm}(G)$ be the right regular representation defined as $\rho(g)(h)=h g^{-1}$ for $g, h \in G$. Then $N=\rho(G)$ is a regular subgroup normalized by $\lambda(G)$, since $\lambda(g) \rho(h) \lambda\left(g^{-1}\right)=\rho(h)$ for all $g, h \in G ; N$ corresponds to a Hopf-Galois structure with $K$-Hopf algebra $H=L[\rho(G)]^{G}=K[G]$, the usual group ring Hopf algebra with its usual action on $L$. Consequently, $\rho(G)$ corresponds to the classical Hopf Galois structure.

Example 3. Again, suppose $L / K$ is Galois with group $G$. Let $N=\lambda(G)$. Then $N$ is a regular subgroup of $\operatorname{Perm}(G)$ which is normalized by $\lambda(G)$, and $N=\rho(G)$ if and only if $N$ abelian. We denote the corresponding Hopf algebra by $H_{\lambda}$. If $G$ is non-abelian, then $\lambda(G)$ corresponds to the canonical non-classical Hopf-Galois structure.

Thus, for $G$ non-abelian there are at least two Hopf-Galois structures on $L / K$. We remark that if $L / K$ is Galois with $G$ simple and non-abelian, then these are the only Hopf Galois structures on $L / K$ [3].

Note that with the classical and the canonical non-classical structures, the regular subgroup $N \leq \operatorname{Perm}(G)$ is isomorphic to $G$. The following example shows that this need not be the case in general.

Example 4. Let $L=\mathbb{Q}(\sqrt{2}, \sqrt{3})$. Then $L / \mathbb{Q}$ is Galois with elementary abelian Galois group $G=\langle r, s\rangle$ with

$$
r(\sqrt{2}+\sqrt{3})=\sqrt{2}-\sqrt{3}, s(\sqrt{2}+\sqrt{3})=-\sqrt{2}+\sqrt{3} .
$$

Let $\eta \in \operatorname{Perm}(G)$ be defined by $\eta\left(r^{i} s^{j}\right)=r^{i-1} s^{i+j-1}$, and let $N=\langle\eta\rangle$. It is routine to verify that $N$ is a regular subgroup of $\operatorname{Perm}(G)$ which is normalized by $\lambda(G)$. Since $N$ is cyclic of order $4, N \neq G$.

\section{The Group $D_{p}$}

Throughout this section, we let $D_{p}$ denote the dihedral group of order $2 p$ for $p$ an odd prime. Explicitly, we write

$$
D_{p}=\left\langle r, s: r^{p}=s^{2}=r s r s=1\right\rangle .
$$

Let $L / K$ be a Galois extension with group $D_{p}$. We shall describe all of the regular subgroups of $\operatorname{Perm}\left(D_{p}\right)$ normalized by $\lambda\left(D_{p}\right)$, and then address the isomorphism questions given in the Introduction. By Examples 2 and 3 we have regular subgroups $\rho\left(D_{p}\right), \lambda\left(D_{p}\right)$ normalized by $\lambda\left(D_{p}\right)$. We construct other regular subgroups of $\operatorname{Perm}\left(D_{p}\right)$.

Lemma 1. Pick $0 \leq c \leq p-1$. Let $\eta_{c}=\lambda(r) \rho\left(r^{c} s\right) \in \operatorname{Perm}\left(D_{p}\right)$, and let $N_{c}=\left\langle\eta_{c}\right\rangle$. Then $N_{c} \cong C_{2 p}$, the cyclic group of order $2 p$, and the $N_{c}$ are distinct as sets. Moreover, $N_{c}$ is a regular subgroup of Perm $\left(D_{p}\right)$ normalized by $\lambda\left(D_{p}\right)$.

Proof. Suppose $0 \leq c \leq p-1$. Since left and right representations commute with each other, $\eta_{c}^{i}=$ $\lambda\left(r^{i}\right) \rho\left(\left(r^{c} s\right)^{i}\right)$. As $|r|=p$ and $\left|r^{c} s\right|=2$ it follows that $\left|N_{c}\right|=2 p$, thus $N_{c} \cong C_{2 p}$. Now suppose $0 \leq d \leq p-1$, $c \neq d$. Since $N_{c} \cong C_{2 p}$, it contains a unique element of order 2, which is $\eta_{c}^{p}=\rho\left(r^{c} s\right)$. Similarly, the unique element of order 2 in $N_{d}$ is $\eta_{d}^{p}=\rho\left(r^{d} s\right)$. Since $c \neq d$, this shows that $N_{c} \neq N_{d}$. 
It remains to show that the stabilizer in $N_{c}$ of any element in $D_{p}$ is trivial, and that $N_{c}$ is normalized by $\lambda\left(D_{p}\right)$. For the remainder of the proof, we write $\eta$ for $\eta_{c}$ and $N$ for $N_{c}$. Let $x \in D_{p}$ and suppose $\eta^{i}[x]=x$. Then

$$
x=\eta^{i}[x]=\lambda\left(r^{i}\right) \rho\left(\left(r^{c} s\right)^{i}\right)[x]=r^{i} x\left(r^{c} s\right)^{-i}
$$

and so,

$$
1_{D_{p}}=x^{-1} r^{i} x\left(r^{c} s\right)^{-i}=r^{ \pm i}\left(r^{c} s\right)^{-i} .
$$

which cannot happen unless $i=0$. Hence $\eta^{i}=1_{N}$ and $N \leq \operatorname{Perm}\left(D_{p}\right)$ is regular.

We now show that $N$ is normalized by $\lambda\left(D_{p}\right)$. Of course, it suffices to show that ${ }^{r} \eta \in N$ and ${ }^{s} \eta \in N$. We have, for $x \in D_{p}$,

$$
\begin{aligned}
& { }^{r} \eta[x]=\lambda(r)\left(\lambda(r) \rho\left(r^{c} s\right)\right) \lambda\left(r^{-1}\right)[x]=r x r^{c} s=\eta[x] \\
& { }^{s} \eta[x]=\lambda(s)\left(\lambda(r) \rho\left(r^{c} s\right)\right) \lambda(s)[x]=\operatorname{srsxr}^{c} s=r^{-1} x r^{c} s=r^{-1} x\left(r^{c} s\right)^{-1}=\eta^{-1}[x] .
\end{aligned}
$$

Thus ${ }^{r} \eta=\eta,{ }^{s} \eta=\eta^{-1}$, and $N$ is normalized by $\lambda\left(D_{p}\right)$.

By [4, Corollary 6.5], the collection $\left\{\rho\left(D_{p}\right), \lambda\left(D_{p}\right), N_{0}, \ldots, N_{p-1}\right\}$ is the complete set of all regular subgroups of Perm $\left(D_{p}\right)$ normalized by $\lambda\left(D_{p}\right)$, hence the corresponding Hopf algebras give all of the HopfGalois structures on $L / K$. We will denote the Hopf algebra corresponding to $N_{c}$ by $H_{c}$ for all $c$.

\section{The Hopf Algebra Isomorphism Questions}

Let $L / K$ be Galois with group $D_{p}$. We have seen that the Hopf algebras which give Hopf-Galois structures on $L / K$ are

$$
\left\{K\left[D_{p}\right], H_{\lambda}, H_{0}, \ldots, H_{p-1} \cdot\right\}
$$

Here, we will investigate when two of these Hopf algebras are isomorphic. Note that throughout this section, when working with Hopf algebras, "isomorphic" refers to isomorphic as Hopf algebras; considering isomorphisms as algebras will be discussed in the next section.

Clearly, $H_{c}$ cannot be isomorphic to either $K\left[D_{p}\right]$ or $H_{\lambda}$ since it is commutative. It remains to determine whether $K\left[D_{p}\right] \cong H_{\lambda}$ or whether $H_{c} \cong H_{d}$ for some $0 \leq c<d \leq p-1$.

Generally, Hopf isomorphism questions reduce to group isomorphism questions.

Proposition 1. Let $L / K$ be a finite separable extension, with Galois closure $E$, and let $G=G a l(E / K)$, $G^{\prime}=\operatorname{Gal}(E / L)$. Let $X=G / G^{\prime}$. Let $N, N^{\prime}$ be regular subgroups of Perm $(X)$ which are normalized by $\lambda(G)$, and let $H, H^{\prime}$ be their corresponding Hopf algebras. If $H \cong H^{\prime}$ then $N \cong N^{\prime}$.

Proof. If $H \cong H^{\prime}$ then $(E[N])^{G} \cong\left(E\left[N^{\prime}\right]\right)^{G}$, hence $E \otimes_{K}(E[N])^{G} \cong E \otimes_{K}\left(E\left[N^{\prime}\right]\right)^{G}$. However $E \otimes_{K}(E[N])^{G} \cong$ $E[N]$ and similarly for $N^{\prime}$, hence $E[N] \cong E\left[N^{\prime}\right]$. Since group algebras (over the same field) are isomorphic as Hopf algebras if and only if their groups are isomorphic, the result follows.

The converse to Proposition 1 is not true as we shall see below in Proposition 4. However, we have:

Proposition 2. Using the notation as in Proposition $1, H \cong H^{\prime}$ if and only if there exists an isomorphism $\phi: N \rightarrow N^{\prime}$ which respects the actions of $G$.

Proof. See [17, Corollary 2.3]. 
We can use this proposition to show that the $H_{c}$ are all isomorphic.

Proposition 3. For $0 \leq c, d \leq p-1$ we have $H_{c} \cong H_{d}$.

Proof. Define $\phi: N_{c} \rightarrow N_{d}$ by $\phi\left(\eta_{c}\right)=\eta_{d}$. This is clearly an isomorphism; it remains to show that it respects the $D_{p}$-actions. But since the $D_{p}$-actions are identical with respect to the generators of the groups this is immediate:

$$
\begin{aligned}
& \phi\left({ }^{r} \eta_{c}\right)=\phi\left(\eta_{c}\right)=\eta_{d}={ }^{r} \eta_{d}={ }^{r} \phi\left(\eta_{c}\right) \\
& \phi\left({ }^{s} \eta_{c}\right)=\phi\left(\eta_{c}^{-1}\right)=\eta_{d}^{-1}={ }^{s} \eta_{d}={ }^{s} \phi\left(\eta_{c}\right)
\end{aligned}
$$

On the other hand, we have:

Proposition 4. $K\left[D_{p}\right] \nRightarrow H_{\lambda}$.

Proof. Suppose $K\left[D_{p}\right] \cong H_{\lambda}$. Then there exists an isomorphism $\phi: \rho\left(D_{p}\right) \rightarrow \lambda\left(D_{p}\right)$ which respects the $D_{p}$-actions. Note that $D_{p}$ acts trivially on $\rho\left(D_{p}\right)$. Pick $1 \leq i \leq p-1$ such that $\phi(\rho(r))=\lambda\left(r^{i}\right)$. Then

$$
\phi\left({ }^{s} \rho(r)\right)=\phi(\rho(r))=\lambda\left(r^{i}\right)
$$

while

$$
{ }^{s} \phi(\rho(r))={ }^{s}\left(\lambda\left(r^{i}\right)\right)=\lambda(s) \lambda\left(r^{i}\right) \lambda(s)=\lambda\left(s r^{i} s\right)=\lambda\left(r^{-i}\right),
$$

which is a contradiction since $r^{i}=r^{-i}$ if and only if $i=0$. Thus, $K\left[D_{p}\right] \not H_{\lambda}$.

Remark 1. One could also prove Proposition 4 as follows. Over $L, K\left[D_{p}\right]$ and $H_{\lambda}$ are isomorphic to $L\left[D_{p}\right]$ as Hopf algebras, thus their duals $K\left[D_{p}\right]^{*}$ and $H_{\lambda}^{*}$ are finite dimensional as algebras over $K$ and separable (as defined in [23, 6.4, page 47]). Using the classification of such $K$-algebras [23, 6.4, Theorem], we conclude that $K\left[D_{p}\right]^{*}$ and $H_{\lambda}^{*}$ are not isomorphic as $K$-Hopf algebras, and so neither are $K\left[D_{p}\right]$ and $H_{\lambda}$. In fact, by [23, 6.3, Theorem], $K\left[D_{p}\right]^{*}$ and $H_{\lambda}^{*}$ are not isomorphic as $K$-algebras, and consequently, $K\left[D_{p}\right]$ and $H_{\lambda}$ are not isomorphic as $K$-coalgebras. As we will show in Section 5, however, $K\left[D_{p}\right] \cong H_{\lambda}$ as $K$-algebras.

Picking $c=0$, we obtain the following.

Theorem 2. There are three K-Hopf algebras which provide Hopf-Galois structures on a dihedral extension $L / K$ of degree 2 p, namely:

1. The group algebra $K\left[D_{p}\right]$, which provides the classical structure

2. The Hopf algebra $H_{\lambda}$, which provides the canonical non-classical structure

3. A commutative $K$-Hopf algebra $H_{0}$ which provides $p$ different structures.

We now wish to consider whether any two of the Hopf algebras above become isomorphic after base change to an intermediate field $K \subset F \subset L$. This question is relatively easy to answer in the dihedral case. Since a $K$-Hopf algebra $H$ is commutative if and only if $F \otimes_{K} H$ is commutative the Hopf algebra $H_{0}$ is not isomorphic to either $K\left[D_{p}\right]$ or $H_{\lambda}$ after base change. What remains is to determine whether we can have $F \otimes_{K} K\left[D_{p}\right] \cong F \otimes_{K} H_{\lambda}$. But this is also easy: Since the center $Z\left(D_{p}\right)$ is trivial, the subgroup of $D_{p}$ defined as $\left\{g \in D_{p}:{ }^{g} \lambda(h)=\lambda(h), \forall h \in D_{p}\right\}$ is trivial. Hence by [13, Corollary 3.2], $F=L$ is the smallest field extension of $L$ for which $F \otimes_{K} H_{\lambda} \cong F\left[D_{p}\right]$. Thus $F=L$ is minimal so that $F \otimes_{K} K\left[D_{p}\right] \cong F \otimes_{K} H_{\lambda}$. 


\section{The Algebra Structure}

Let $L / K$ be Galois with group $D_{p}, \mathbb{Q} \subseteq K$. In this section we investigate the question of when two Hopf algebras providing Hopf-Galois structures on $L / K$ are isomorphic as algebras. Throughout this section, when working with Hopf algebras, "isomorphic" refers to isomorphic as algebras. Since char $(K)$ does not divide $[L: K]$, Maschke's theorem and a result of Amitsur [1, Theorem 1] shows that the Hopf algebras are left semisimple.

Of course, $H_{c} \cong H_{d}$ for all $0 \leq c, d \leq p-1$, and these (Hopf) algebras are not isomorphic to either $K\left[D_{p}\right]$ or $H_{\lambda}$ since they are commutative; they remain non-isomorphic after base change for the same reason.

It remains to consider the classical and the canonical non-classical structures, $K\left[D_{p}\right]$ and $H_{\lambda}$, respectively. Our main tool will be the Wedderburn-Artin decomposition.

The decomposition of $\mathbb{Q}\left[D_{p}\right]$ is given in [11, Example (7.39)].

Theorem 3. Let $D_{p}, p \geq 3$ prime, be the dihedral group of order $2 p$. Then

$$
\mathbb{Q}\left[D_{p}\right] \cong \mathbb{Q} \times \mathbb{Q} \times \operatorname{Mat}_{2}\left(\mathbb{Q}\left(\zeta_{p}+\zeta_{p}^{-1}\right)\right)
$$

where $\zeta_{p}$ denotes a primitive pth root of unity.

Let $E=K \cap \mathbb{Q}\left(\zeta_{p}+\zeta_{p}^{-1}\right)$ and $l=\left[\mathbb{Q}\left(\zeta_{p}+\zeta_{p}^{-1}\right): E\right]$. Then

$$
K\left[D_{p}\right] \cong \begin{cases}K \times K \times\left(\operatorname{Mat}_{2}\left(K\left(\zeta_{p}+\zeta_{p}^{-1}\right)\right)\right)^{(p-1) /(2 l)} & \zeta_{p}+\zeta_{p}^{-1} \notin K \\ K \times K \times\left(\operatorname{Mat}_{2}(K)\right)^{(p-1) / 2} & \zeta_{p}+\zeta_{p}^{-1} \in K\end{cases}
$$

The former case follows by noting that $E=\mathbb{Q}(\alpha)$ for $\alpha \in E$ satisfying an irreducible monic polynomial of degree $(p-1) /(2 l)$ over $\mathbb{Q}$. The latter case follows from observing that if $\zeta_{p}+\zeta_{p}^{-1} \in K$, then the $(p-1) / 2$ 2-dimensional irreducible representations of $D_{p}$ over $\mathbb{C}$ correspond to $(p-1) / 2$ characters with values in $K$ [22, Chapter 5, 5.3].

What can be said about the decomposition of $H_{\lambda}$ ? Since $H_{\lambda}$ is left semisimple, the $K$-algebra $H_{\lambda}$ decomposes into a product of matrix rings over division rings,

$$
H_{\lambda} \cong \operatorname{Mat}_{q_{1}}\left(R_{1}\right) \times \operatorname{Mat}_{q_{2}}\left(R_{2}\right) \times \cdots \times \operatorname{Mat}_{q_{t}}\left(R_{t}\right) .
$$

The division rings $R_{i}$ are finite dimensional $K$-algebras.

Now, there are exactly two 1-dimensional irreducible representations of $D_{p}$, with characters $\chi_{1}$ and $\chi_{2}$, corresponding to mutually orthogonal idempotents

$$
e_{1}=\frac{1}{2 p} \sum_{g \in D_{p}} \chi_{1}\left(g^{-1}\right) g, \quad e_{2}=\frac{1}{2 p} \sum_{g \in D_{p}} \chi_{2}\left(g^{-1}\right) g
$$

in $L\left[D_{p}\right]$. Both $e_{1}$ and $e_{2}$ are fixed by the action of $D_{p}$, hence $e_{1}, e_{2} \in H_{\lambda}$. It follows that

$$
H_{\lambda} \cong K \times K \times \prod_{j=1}^{m} \operatorname{Mat}_{q_{j}}\left(R_{j}\right)
$$


where $q_{j} \geq 1$ are integers and $R_{j}$ are division rings. For later use, we set $S_{j}=\operatorname{Mat}_{q_{j}}\left(R_{j}\right)$ for $1 \leq j \leq m$. Observe that

$$
\sum_{j=1}^{m} \operatorname{dim}_{K}\left(R_{j}\right) \cdot q_{j}^{2}=2(p-1) .
$$

For the moment we assume $p=3$, so that $\sum_{j=1}^{m} \operatorname{dim}_{K}\left(R_{j}\right) \cdot q_{j}^{2}=4$. Since the dimension of a division algebra over its center is a perfect square, we conclude that

$$
H_{\lambda} \cong K \times K \times \operatorname{Mat}_{q}(R),
$$

where $1 \leq q \leq 2$ and $R$ is a division ring. If $q=1$, then the corresponding division ring $R$ is non-commutative. If $q=2$, then $R=K$.

Assume that the base field $K=\mathbb{Q}$, and let $L$ be the splitting field of $x^{3}-v$ over $\mathbb{Q}$ where $v$ is not a 3rd power in $\mathbb{Q}$. Let $\zeta_{3}$ denote a primitive 3rd root of unity and let $\alpha=\sqrt[3]{v}$. Then $L=\mathbb{Q}\left(\alpha, \zeta_{3}\right)$ is Galois with group $D_{3}$. The Galois action is given as $r(\alpha)=\zeta_{3} \alpha, r\left(\zeta_{3}\right)=\zeta_{3}, s(\alpha)=\alpha, s\left(\zeta_{3}\right)=\zeta_{3}^{2}$. Let

$$
b=\alpha s+\alpha \zeta_{3} s r+\alpha \zeta_{3}^{2} s r^{2} .
$$

Then $b \in L\left[D_{3}\right]$, and since $b$ is fixed by all elements of $D_{3}, b \in H_{\lambda}$. Moreover, direct computation yields $b^{2}=0$. Thus the only possibility is that $q=2$ in (3), and so,

$$
H_{\lambda} \cong \mathbb{Q} \times \mathbb{Q} \times \operatorname{Mat}_{2}(\mathbb{Q}) .
$$

Since $\mathbb{Q}\left[D_{3}\right] \cong \mathbb{Q} \times \mathbb{Q} \times \operatorname{Mat}_{2}(\mathbb{Q})$ by Theorem 3, here we have an instance of $H_{\lambda} \cong \mathbb{Q}\left[D_{3}\right]$ as $\mathbb{Q}$-algebras. Surprisingly, this is true for any $L / K$ Galois with group $D_{p}, p \geq 3$. In fact, it holds even more generally:

Theorem 4 (Greither). Let $\mathbb{Q} \subseteq K$, and let $L / K$ be a Galois extension with group $G$. Then

$$
H_{\lambda} \cong K[G]
$$

as $K$-algebras.

Proof. We prove the special case where $G=D_{p}$. We thank C. Greither for the method of proof.

From (1) we obtain

$$
L \otimes_{K} H_{\lambda} \cong L \otimes_{K} K\left[D_{p}\right] \cong L\left[D_{p}\right] \cong \begin{cases}L \times L \times\left(\operatorname{Mat}_{2}\left(L \otimes_{K} K\left(\zeta_{p}+\zeta_{p}^{-1}\right)\right)\right)^{(p-1) /(2 l)} & \zeta_{p}+\zeta_{p}^{-1} \notin K \\ L \times L \times\left(\operatorname{Mat}_{2}(L)\right)^{(p-1) / 2} & \zeta_{p}+\zeta_{p}^{-1} \in K\end{cases}
$$

with $l=\left[\mathbb{Q}\left(\zeta_{p}+\zeta_{p}^{-1}\right): E\right]=\left[K\left(\zeta_{p}+\zeta_{p}^{-1}\right): K\right], E=K \cap \mathbb{Q}\left(\zeta_{p}+\zeta_{p}^{-1}\right)$. Thus the decomposition of $L\left[D_{p}\right]$ contains components of the form $L$ and $\operatorname{Mat}_{2}\left(L \otimes_{K} F\right)$, with $F=K\left(\zeta_{p}+\zeta_{p}^{-1}\right)$.

The Hopf algebra $H_{\lambda}$ descends from $L\left[D_{p}\right]$ via the action of $D_{p}$ as usual: an element of $D_{p}$ acts by conjugation on $D_{p}$ and by the Galois action on $L$. A character of $D_{p}$ has the same value on conjugate elements in $D_{p}$ [22, Chapter 2, 2.1, Proposition 1(iii)], and so the central indecomposable idempotents of $\mathbb{C}\left[D_{p}\right]$ as constructed in [22, Chapter 6,6.3, Exercise 6.4] are fixed by conjugation by elements of $D_{p}$. Let $e$ be a central indecomposable idempotent in $K\left[D_{p}\right]$. Since $e$ is in the center of $\mathbb{C}\left[D_{p}\right], e$ is a sum of central indecomposable idempotents of $\mathbb{C}\left[D_{p}\right][22$, Chapter 6, 6.3, Exercise 6.4]. Thus, conjugation by elements of $D_{p}$ fixes $e$. 
Now, the central indecomposable idempotents of $K\left[D_{p}\right]$ correspond to the components in the decomposition (1); let $M$ be the component of $K\left[D_{p}\right]$ corresponding to $e$. For $\alpha \in M, g \in D_{p}, g \alpha g^{-1}=g \alpha e g^{-1}=$ $g \alpha g^{-1} g e g^{-1}=g \alpha g^{-1} e \in M$, and so, congugation by $g$, which is an automorphism of $K\left[D_{p}\right]$, restricts to an automorphism of each component of $K\left[D_{p}\right]$. Hence the action of $D_{p}$ preserves the components in the decomposition of $L \otimes_{K} K\left[D_{p}\right] \cong L\left[D_{p}\right]$. So $D_{p}$ can be thought of as acting on these components. The two copies of $L$ in the decomposition of $L\left[D_{p}\right]$ descend to the two copies of $K$ in the decomposition (2) of $H_{\lambda}$, and each copy of $\operatorname{Mat}_{2}\left(L \otimes_{K} F\right)$ descends to a component $S=S_{j}$ in the decomposition (2) of $H_{\lambda}$; the $K$-algebra $S$ is an $L$-form of $\operatorname{Mat}_{2}(F)$. We want to show that $S \cong \operatorname{Mat}_{2}(F)$ as $K$-algebras, and so $H_{\lambda} \cong K\left[D_{p}\right]$.

Let $\operatorname{Aut}\left(\operatorname{Mat}_{2}(F)\right)$ denote the automorphism group scheme of $\operatorname{Mat}_{2}(F)$ in the sense of [23, §7.6]. By [23, Theorem, p. 137] the isomorphism classes of $L$-forms of $\operatorname{Mat}_{2}(F)$ correspond to the cohomology set $\mathrm{H}^{1}\left(D_{p}\right.$, Aut $\left(\operatorname{Mat}_{2}\left(L \otimes_{K} F\right)\right)$. A 1-cocycle (crossed homomorphism) is a function $f: D_{p} \rightarrow \operatorname{Aut}\left(\operatorname{Mat}_{2}\left(L \otimes_{K}\right.\right.$ $F)$ ) which satisfies $f(g h)=f(g) \circ(g \cdot f(h))$, for $g, h \in D_{p}$. The action $g \cdot f(h)$ of the element $g \in D_{p}$ on the automorphism $f(h)$ in $\operatorname{Aut}\left(\operatorname{Mat}_{2}\left(L \otimes_{K} F\right)\right)$ is induced by the Galois action on $L$ : we have

$$
g \cdot f(h)=\left(g \otimes I_{F}\right) f(h)\left(g^{-1} \otimes I_{F}\right),
$$

where $g, h \in D_{p}$, and $I_{F}$ is the identity map on $F$. The trivial element in $\mathrm{H}^{1}\left(D_{p}, \operatorname{Aut}\left(\operatorname{Mat}_{2}\left(L \otimes_{K} F\right)\right)\right.$ is represented by the 1 -cocycle

$$
g \mapsto\left(g \otimes I_{F}\right) \phi\left(g^{-1} \otimes I_{F}\right) \phi^{-1},
$$

where $\phi$ is any element of $\operatorname{Aut}\left(\operatorname{Mat}_{2}\left(L \otimes_{K} F\right)\right)$. The $L$-form $S$ comes from a particularly simple 1-cocycle $\hat{f}: D_{p} \rightarrow \operatorname{Aut}\left(\operatorname{Mat}_{2}\left(L \otimes_{K} F\right)\right)$. For $g \in D_{p}, \hat{f}(g)$ is conjugation by $g$ on $\operatorname{Mat}_{2}(F) \subseteq \operatorname{Mat}_{2}\left(L \otimes_{K} F\right)$. Let $[\hat{f}]$ denote the class of $\hat{f}$ in $\mathrm{H}^{1}\left(D_{p}\right.$, Aut $\left(\operatorname{Mat}_{2}\left(L \otimes_{K} F\right)\right)$.

Let Inn $\left(\operatorname{Mat}_{2}\left(L \otimes_{K} F\right)\right)$ denote the group of inner automorphisms. Since every element of $\operatorname{Inn}\left(\operatorname{Mat}_{2}\left(L \otimes_{K}\right.\right.$ $F)$ ) is given as conjugation by some element of $\mathrm{GL}_{2}\left(L \otimes_{K} F\right)$, there is a surjection of groups $\psi: \mathrm{GL}_{2}\left(L \otimes_{K}\right.$ $F) \rightarrow \operatorname{Inn}\left(\operatorname{Mat}_{2}\left(L \otimes_{K} F\right)\right)$ with $\operatorname{ker}(\psi)=\left(L \otimes_{K} F\right)^{\times}$. Thus, there is an induced map in cohomology

$$
\mathrm{H}^{1}\left(D_{p}, \mathrm{GL}_{2}\left(L \otimes_{K} F\right)\right) \stackrel{\psi}{\longrightarrow} \mathrm{H}^{1}\left(D_{p}, \operatorname{Inn}\left(\operatorname{Mat}_{2}\left(L \otimes_{K} F\right)\right)\right) .
$$

A 1-cocycle class $[q] \in \mathrm{H}^{1}\left(D_{p}, \mathrm{GL}_{2}\left(L \otimes_{K} F\right)\right)$ is represented by a function $q: D_{p} \rightarrow \mathrm{GL}_{2}\left(L \otimes_{K} F\right)$ which satisfies $q(g h)=q(g)(g \cdot q(h))$. The action of $g \in D_{p}$ on $q(h) \in \mathrm{GL}_{2}\left(L \otimes_{K} F\right)$ is through the Galois action on $L$ as above.

There is a special cocycle class $[\hat{q}] \in \mathrm{H}^{1}\left(D_{p}, \mathrm{GL}_{2}\left(L \otimes_{K} F\right)\right)$ represented by the function $\hat{q}$ in which each $g \in D_{p}$ is identified with its image in $\operatorname{Mat}_{2}(F) \subseteq \operatorname{Mat}_{2}\left(L \otimes_{K} F\right)$ under the map $K\left[D_{p}\right] \rightarrow \operatorname{Mat}_{2}(F)$. So conjugation by $g$ in $\operatorname{Mat}_{2}(F)$ is precisely the conjugation action of $g$ on $K\left[D_{p}\right]$ restricted to the component $\operatorname{Mat}_{2}(F)$. It follows that

$$
\psi([\hat{q}])=[\hat{f}] .
$$

The class $[\hat{f}]$ corresponds to the isomorphism class of the $L$-form $S$.

Now if $\zeta_{p}+\zeta_{p}^{-1} \in K$, then $F=K$. Thus, $L=L \otimes_{K} F$ and

$$
\mathrm{H}^{1}\left(D_{p}, \mathrm{GL}_{2}\left(L \otimes_{K} F\right)\right)=\mathrm{H}^{1}\left(D_{p}, \mathrm{GL}_{2}(L)\right) .
$$

By Hilbert's Theorem $90, \mathrm{H}^{1}\left(D_{p}, \mathrm{GL}_{2}(L)\right)$ is trivial, and so, $[\hat{q}]$ is trivial, and consequently, $[\hat{f}]$ is trivial. It follows that $S \cong \operatorname{Mat}_{2}(K)$ as $K$-algebras, and so $H_{\lambda} \cong K\left[D_{p}\right]$. 
If $\zeta_{p}+\zeta_{p}^{-1} \notin K$, then C. Greither has provided a generalization of Hilbert's Theorem 90 to yield $\mathrm{H}^{1}\left(D_{p}, \mathrm{GL}_{2}\left(L \otimes_{K} F\right)\right.$ trivial. As above, $[\hat{f}]$ is trivial, and so $S \cong \operatorname{Mat}_{2}(F)$ as $K$-algebras. It follows that $H_{\lambda} \cong K\left[D_{p}\right]$.

We summarize our findings in this section. The Hopf algebras that provide Hopf-Galois structures in the case that $L / K$ is Galois with group $D_{p}$ fall into two $K$-algebra isomorphism classes represented by $K\left[D_{p}\right]$ and $H_{0}$. So a single $K$-algebra (e.g., $K\left[D_{p}\right]$ ) can be be endowed with multiple coalgebra structures, resulting in multiple (non-isomorphic) Hopf algebras (e.g., $K\left[D_{p}\right], H_{\lambda}$ ) giving different Hopf-Galois structures on $L / K$ (e.g., classical and canonical non-classical).

\section{Explicit Structure Computations}

Let $L / K$ be Galois with group $D_{p}, \mathbb{Q} \subseteq K$. We find generators over $K$ for the Hopf algebras $K\left[D_{p}\right], H_{\lambda}$, $H_{0}, H_{1}, \ldots, H_{p-1}$ constructed above. Let $L^{\langle r\rangle}$ be the unique quadratic extension of $K$ contained in $L$. Pick $d \in L$ such that $L^{\langle r\rangle}=K(\sqrt{d})$. Note that $s(\sqrt{d})=-\sqrt{d}$. Additionally, let $y \in L$ be so that $K(y)=L^{\langle s\rangle}$.

The simplest case, of course, is $K\left[D_{p}\right]$ : it has a $K$-basis $D_{p}$, and $\{r, s\}$ generates $K\left[D_{p}\right]$ as a $K$-algebra.

We next turn to $H_{\lambda}$. Suppose $h \in H_{\lambda}$. Identifying $D_{p}$ with $\lambda\left(D_{p}\right)$, we have $h \in L\left[D_{p}\right]$ with $h$ fixed by $D_{p}$. Let

$$
h=\sum_{i=0}^{p-1} a_{i} r^{i}+\sum_{i=0}^{p-1} b_{i} r^{i} s, a_{i}, b_{i} \in L
$$

Then

$$
{ }^{r} h=\sum_{i=0}^{p-1} r\left(a_{i}\right) r^{i}+\sum_{i=0}^{p-1} r\left(b_{i}\right) r^{i+1} s r^{-1}=\sum_{i=0}^{p-1} r\left(a_{i}\right) r^{i}+\sum_{i=0}^{p-1} r\left(b_{i}\right) r^{i+2} s,
$$

and since ${ }^{r} h=h$ we have $a_{i} \in L^{\langle r\rangle}$ and $r\left(b_{i}\right)=b_{i+2}$ for all $i$ (where $i+2$ is considered $\bmod p$ ). Thus, $b_{i}=r^{i(p+1) / 2}\left(b_{0}\right)$ for all $i$.

Furthermore,

$$
s h=\sum_{i=0}^{p-1} s\left(a_{i}\right) s r^{i} s+\sum_{i=0}^{p-1} s\left(b_{i}\right) s r^{i} s^{2}=\sum_{i=0}^{p-1} s\left(a_{i}\right) r^{-i}+\sum_{i=0}^{p-1} s\left(b_{i}\right) r^{-i} s,
$$

which after interchanging $i$ with $p-i$ for all $i \neq 0$ gives

$$
{ }^{s} h=s\left(a_{0}\right)+s\left(b_{0}\right) s+\sum_{i=1}^{p-1} s\left(a_{p-i}\right) r^{i}+\sum_{i=1}^{p-1} s\left(b_{p-i}\right) r^{i} s
$$

and so $a_{0} \in K, s\left(a_{i}\right)=a_{p-i}, s\left(b_{0}\right)=b_{0}$. Note that $s\left(b_{i}\right)=b_{p-i}$ as well, but this followed previously since

$$
s\left(b_{i}\right)=s r^{i(p+1) / 2}\left(b_{0}\right)=r^{-i(p+1) / 2} s\left(b_{0}\right)=r^{-i(p+1) / 2}\left(b_{0}\right)=r^{(p-i)(p+1) / 2}\left(b_{0}\right)=b_{p-i} .
$$

Since $r^{(p-i)(p+1) / 2}=r^{i(p-1) / 2}$ it follows that

$$
H_{\lambda}=\left\{a_{0}+\sum_{i=1}^{(p-1) / 2}\left(a_{i} r^{i}+s\left(a_{i}\right) r^{-i}\right)+b_{0} s+\sum_{i=1}^{p-1} r^{i(p-1) / 2}\left(b_{0}\right) r^{-i} s: a_{0} \in K, a_{i} \in L^{\langle r\rangle}, b_{0} \in L^{\langle s\rangle}\right\} .
$$


Example 5. Suppose $p=3$. Then

$$
H_{\lambda}=\left\{a_{0}+a_{1} r+s\left(a_{1}\right) r^{2}+b_{0} s+r\left(b_{0}\right) s r+r^{2}\left(b_{0}\right) s r^{2}: a_{0} \in K, a_{1} \in L^{\langle r\rangle}, b_{0} \in L^{\langle s\rangle}\right\} .
$$

A $K$-basis for $H_{\lambda}$ is

$$
\left\{1, r+r^{2}, \sqrt{d}\left(r-r^{2}\right), s+r s+r^{2} s, y s+r(y) r s+r^{2}(y) r^{2} s, y^{2} s+r\left(y^{2}\right) r s+r^{2}\left(y^{2}\right) r^{2} s\right\} .
$$

We next consider the structure of $H_{c}, 0 \leq c \leq p-1$. If we write

$$
h=\sum_{i=0}^{2 p-1} a_{i} \eta^{i}, \quad \eta=\eta_{c}
$$

where $a_{i} \in L$ for all $i$ then, since $r$ acts trivially on $\eta$,

$$
{ }^{r} h=\sum_{i=0}^{2 p-1} r\left(a_{i}\right) \eta^{i}=\sum_{i=0}^{2 p-1} a_{i} \eta^{i}
$$

Thus $r\left(a_{i}\right)=a_{i}$ for all $i$, hence $a_{i} \in L^{\langle r\rangle}$. Also, since ${ }^{s} \eta=\eta^{-1}$,

$$
{ }^{s} h=\sum_{i=0}^{2 p-1} s\left(a_{i}\right) \eta^{-i}=\sum_{i=0}^{2 p-1} a_{i} \eta^{i}
$$

from which it follows that $s\left(a_{0}\right)=a_{0}$ and $s\left(a_{i}\right)=a_{2 p-i}$ for all $i>0$. In particular, $s\left(a_{p}\right)=a_{p}$, so $a_{0}, a_{p} \in K$. Thus,

$$
H_{c}=\left\{a_{0}+a_{p} \eta^{p}+\sum_{i=1}^{p-1}\left(a_{i} \eta^{i}+s\left(a_{i}\right) \eta^{-i}\right): a_{0}, a_{p} \in K, a_{i} \in L^{\langle r\rangle}, 1 \leq i \leq p-1\right\},
$$

and $H_{c}$ has $K$-basis

$$
\left\{1, \eta^{p}, \eta+\eta^{-1}, \eta^{2}+\eta^{-2}, \ldots, \eta^{p-1}+\eta^{-(p-1)}, \sqrt{d}\left(\eta-\eta^{-1}\right), \sqrt{d}\left(\eta^{2}-\eta^{-2}\right), \ldots, \sqrt{d}\left(\eta^{p-1}-\eta^{-(p-1)}\right)\right\} .
$$

Example 6. Suppose $p=3$. Then

$$
H_{c}=\left\{a_{0}+a_{3} \eta^{3}+a_{1} \eta+s\left(a_{1}\right) \eta^{5}+a_{2} \eta^{2}+s\left(a_{2}\right) \eta^{4}: a_{0}, a_{3} \in K, a_{1}, a_{2} \in L^{\langle r\rangle}\right\},
$$

and $H_{c}$ has $K$-basis

$$
\left\{1, \eta^{3}, \eta+\eta^{5}, \eta^{2}+\eta^{4}, \sqrt{d}\left(\eta-\eta^{5}\right), \sqrt{d}\left(\eta^{2}-\eta^{4}\right)\right\}
$$

\section{Example: Hopf Galois Structures in the Case $D_{3}$}

We close with an analysis of the Hopf Galois structures in the case $p=3$. Let $L / K$ be any Galois extension with group $D_{3}, \mathbb{Q} \subseteq K$. As we have seen, there are two regular subgroups normalized by $\lambda\left(D_{3}\right)$ and isomorphic to $D_{3}$, namely, $\rho\left(D_{3}\right)$ and $\lambda\left(D_{3}\right)$, and three regular subgroups normalized by $\lambda\left(D_{3}\right)$ and isomorphic to $C_{6}$, the cyclic group of order 6, namely, $N_{0}, N_{1}$ and $N_{2}$. 
By Proposition $4 K\left[D_{3}\right] \nRightarrow H_{\lambda}$, as $K$-Hopf algebras, and by Theorem $4 K\left[D_{3}\right] \cong H_{\lambda}$, as $K$-algebras, with Wedderburn-Artin decomposition

$$
K\left[D_{3}\right] \cong H_{\lambda} \cong K \times K \times \operatorname{Mat}_{2}(K) .
$$

By Proposition 3, $H_{0} \cong H_{1} \cong H_{2}$ as Hopf algebras, and hence as $K$-algebras.

We seek the Wedderburn-Artin decomposition and the Hopf algebra structure of $H_{0}$ (hence of $H_{1}$ and $H_{2}$ ). In contrast to the situation with $H_{\lambda}$, the structure of $H_{0}$ seems to depend on the extension $L / K$, specifically on the fixed field $L^{\langle r\rangle}$.

Here is how we can compute the structure of $H_{0}$. By [20, Corollary 3.6],

$$
\left\{g \in \lambda\left(D_{3}\right):{ }^{g} \eta=\eta, \forall \eta \in N_{0}\right\}
$$

is precisely the 3-Sylow subgroup $\lambda(\langle r\rangle) \leq \lambda\left(D_{3}\right)$, which we identify with $\langle r\rangle$. There is an induced action of $D_{3} /\langle r\rangle$ on $L\left[N_{0}\right]$. Note that $D_{3} /\langle r\rangle \cong C_{2}$, the cyclic group of order 2. By the Fundamental Theorem of Galois theory, $D_{3} /\langle r\rangle \cong C_{2}$ is the group of the Galois extension $F / K$, where $F=L^{\langle r\rangle} ; F$ is a quadratic extension of $K$. We write $F=K[z] /\left(z^{2}-b\right)$ for $b \in K, z$ indeterminate.

Now, $D_{3} /\langle r\rangle \cong C_{2}$ can be viewed as the group of automorphisms of $N_{0} \cong C_{6}$. We have

$$
H_{0}=\left(L\left[N_{0}\right]\right)^{D_{3}}=\left(F\left[C_{6}\right]\right)^{C_{2}},
$$

where the action of $C_{2}$ on $F\left[C_{6}\right]$ is by the Galois group on $F$ and as automorphisms on $C_{6}$.

Now, $F$ is a $C_{2}$-Galois extension of $K$, [14, page 130]. So by [14, Theorem 5], $F$ corresponds to an $F$-Hopf algebra form of $K\left[C_{6}\right]$, namely, $\left(F\left[C_{6}\right]\right)^{C_{2}}$, which must of course be $H_{0}$.

And so, $H_{0}$ is the fixed ring of $F\left[C_{6}\right]$ under the action of $C_{2}$, and $H_{0}$ is an $F$-form of $K\left[C_{6}\right]$. Under these conditions, $H_{0}$ can be characterized. Let $x, y$ be indeterminates and recall $F=K[z] /\left(z^{2}-b\right)$. The method of Haggenmüller and Pareigis in [14, Theorem 6, p. 134] applies to yield $H_{0} \cong K[x, y] / I$,

$$
I=\left(y^{2}-b x^{2}+u,(x-2)(x-1)(x+1)(x+2),(x-1)(x+1)(x y)\right),
$$

with $u \in K^{\times}, u=4 b$. The Hopf algebra structure of $H_{0}$ is defined by

$$
\begin{aligned}
\Delta(\bar{x}) & =\frac{1}{2} \bar{x} \otimes \bar{x}+\frac{1}{2 b} \bar{y} \otimes \bar{y}, \\
\Delta(\bar{y}) & =\frac{1}{2} \bar{x} \otimes \bar{y}+\frac{1}{2} \bar{y} \otimes \bar{x}, \\
\varepsilon(\bar{x})=2, \quad \varepsilon(\bar{y}) & =0, \quad \sigma(\bar{x})=\bar{x}, \quad \sigma(\bar{y})=-\bar{y} .
\end{aligned}
$$

where $\bar{x}=x \bmod I, \bar{y}=y \bmod I$.

Remark 2. The Hopf algebra structure of $H_{0}$ does not depend on the choice of generator for the quadratic extension $F$. Indeed, suppose that $F=F^{\prime}$ where $F^{\prime}=K[z] /\left(\left(z^{2}-a^{\prime} z-b^{\prime}\right)\right.$. Then the induced isomorphism $\phi: F\left[C_{6}\right] \rightarrow F^{\prime}\left[C_{6}\right]$ respects the action of $C_{2}$, hence the fixed rings $H_{0}, H_{0}^{\prime}$ are isomorphic as $K$-Hopf algebras.

Example 7. We assume that the base field $K=\mathbb{Q}$ and compute the structure of $H_{0}$ in the case that $L$ is the splitting field of $x^{3}-v$, irreducible over $\mathbb{Q}$. In this case, $F=L^{\langle r\rangle}=\mathbb{Q}\left(\zeta_{3}\right)$, hence $F=\mathbb{Q}[z] /\left(z^{2}+3\right)$, and so, $b=-3, u=-12$. We then have $H_{0}=\mathbb{Q}[x, y] / I$, with

$$
I=\left(y^{2}+3 x^{2}-12,(x-2)(x-1)(x+1)(x+2),(x-1)(x+1)(x y)\right) .
$$


The Hopf algebra structure of $H_{0}$ is given as

$$
\begin{aligned}
\Delta(\bar{x}) & =\frac{1}{2} \bar{x} \otimes \bar{x}-\frac{1}{6} \bar{y} \otimes \bar{y}, \\
\Delta(\bar{y}) & =\frac{1}{2} \bar{x} \otimes \bar{y}+\frac{1}{2} \bar{y} \otimes \bar{x} \\
\varepsilon(\bar{x})=2, \quad \varepsilon(\bar{y}) & =0, \quad \sigma(\bar{x})=\bar{x}, \quad \sigma(\bar{y})=-\bar{y} .
\end{aligned}
$$

We can also obtain the Wedderburn-Artin decomposition of $H_{0}$ in Example 7.

Proposition 5. Assume the conditions of Example 7. Then

$$
H_{0} \cong \mathbb{Q} \times \mathbb{Q} \times \mathbb{Q} \times \mathbb{Q} \times \mathbb{Q} \times \mathbb{Q},
$$

as $\mathbb{Q}$-algebras.

Proof. The ideal $I$ determines an affine variety in $X \subseteq \mathbb{Q}^{2}$ consisting of exactly six points:

$$
P_{1}=(-2,0), P_{2}=(-1,3), P_{3}=(1,3), P_{4}=(2,0), P_{5}=(1,-3), P_{6}=(-1,-3),
$$

This is the set of common zeros of the polynomials in $I$, see Fig. 1 .

As a commutative $\mathbb{Q}$-algebra, $H_{0} \cong \mathbb{Q}[x, y] / I$ is a product of fields with $\operatorname{dim}_{\mathbb{Q}}\left(H_{0}\right)=6$. For $1 \leq j \leq 6$, let $\left(x_{j}, y_{j}\right)$ be the coordinates of the point $P_{j}$ and let $\Psi_{j}: H_{0} \rightarrow \mathbb{Q}$ denote the ring homomorphism defined as $\bar{x} \mapsto x_{j}, \bar{y} \mapsto y_{j}$. Then $\Psi_{j}$ is surjective and $\operatorname{ker}\left(\Psi_{j}\right)$ is an ideal $I_{j}$ of $H_{0}$ of dimension 5 over $\mathbb{Q}$. Now, $\bar{y}-y_{j}-6 \bar{x}+6 x_{j} \in I_{j}$, yet $\bar{y}-y_{j}-6 \bar{x}+6 x_{j} \notin I_{k}$ whenever $j \neq k$. Thus the ideals $I_{j}, 1 \leq j \leq 6$, are distinct

Fig. 1 Graph of variety deter-

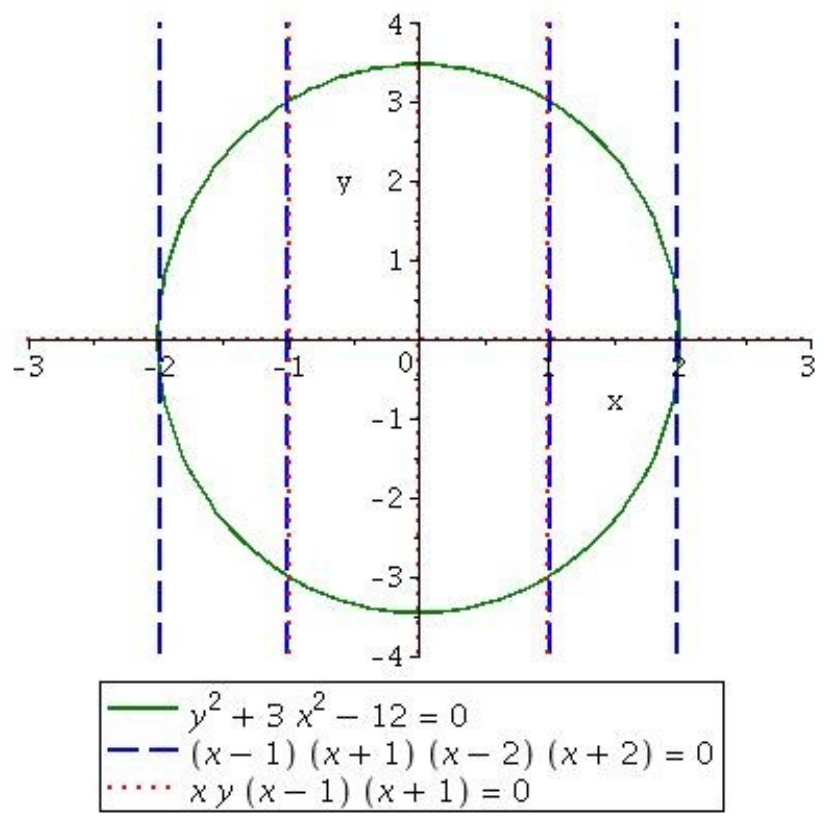
mined by $I$. 
and therefore must arise by omitting one factor isomorphic to $\mathbb{Q}$ from the Wedderburn-Artin decomposition of $H_{0}$. It follows that the decomposition of $H_{0}$ must contain at least 6 factors isomorphic to $\mathbb{Q}$, hence $H_{0} \cong \mathbb{Q}^{6}$.

Acknowledgements The authors would like to thank the referee for comments and suggestions which improved the exposition and content of this paper.

\section{References}

1. S. A. Amitsur. The Radical of field extensions. Bull. Res. Council Israel, Sect. F, 7F(1), 1-10, 1957/1958.

2. N. P. Byott. Uniqueness of Hopf Galois structure of separable field extensions, Comm. Algebra, 24(10), 3217-3228, 1996.

3. N. P. Byott. Hopf Galois structures on field extensions with simple Galois groups. Bull. London. Math. Soc., 36, 23-29, 2004.

4. N. P. Byott. Hopf-Galois structures on Galois field extensions of degree pq. J. Pure Appl. Algebra, 188(1 - 3), 45-57, 2004.

5. S. Carnahan and L. Childs. Counting Hopf Galois structures on non-abelian Galois field extensions. J. Algebra, 218(1), 81-92, 1999.

6. S. U. Chase and M. E. Sweedler. Hopf algebras and Galois theory. Lecture Notes in Mathematics, Vol. 97. SpringerVerlag, Berlin, 1969.

7. L. N. Childs. Taming Wild Extensions: Hopf Algebras and Local Galois Module Theory. AMS: Mathematical Surveys and Monographs, 80, 2000.

8. L. N. Childs. Some Hopf Galois structures arising from elementary abelian p-groups. Proc. Amer. Math. Soc., 135(11), 3453-3460, 2007.

9. L. N. Childs. Hopf Galois structures on Kummer extensions of prime power degree New York J. Math., 17, 51-74, 2011.

10. L. N. Childs. Fixed-Point free endomorphisms and Hopf Galois structures. Proc. Amer. Math. Soc., 141(4), 1255-1265, 2013.

11. C. W. Curtis and I. Reiner. Methods of representation theory. Vol. I. John Wiley \& Sons, Inc., New York, 1981.

12. S. C. Featherstonhaugh, A. Caranti, and L. N. Childs. Abelian Hopf Galois structures on prime-power Galois field extensions. Trans. Amer. Math. Soc., 364(7), 3675-3684, 2012.

13. C. Greither and B. Pareigis. Hopf Galois theory for separable field extensions. J. Algebra, 106(1), 239-258, 1987.

14. R. Haggenmüller, B. Pareigis, Hopf algebra forms on the multiplicative group and other groups, manuscripta math., 55, 121-135, 1986.

15. N. Jacobson. Galois theory of purely inseparable fields of exponent one. Amer. J. Math., 66, 645-648, 1944.

16. A. Koch. Scaffolds and integral Hopf Galois module structure on purely inseparable extensions. New York J. Math., 21, 73-91, 2015.

17. A. Koch, T. Kohl, P. Truman, and R. Underwood. Isomorphism problems for Hopf-Galois structures on separable field extensions. arXiv: 1711.05554, 2017.

18. T. Kohl. Classification of the Hopf Galois structures on prime power radical extensions. J. Algebra, 207(2), 525-546, 1998.

19. T. Kohl. Groups of order 4p, twisted wreath products and Hopf-Galois theory. J. Algebra, 314(1), 42-74, 2007.

20. T. Kohl. Regular permutation groups of order $m p$ and Hopf Galois structures. Algebra Number Theory, 7(9), 2203-2240, 2013.

21. T. Kohl. Hopf-Galois structures arising from groups with unique subgroup of order p. Algebra Number Theory, 10(1), 37-59, 2016.

22. J.-P. Serre. Linear Representations of Finite Groups. Springer-Verlag, New York, 1977.

23. W. C. Waterhouse. Introduction to Affine Group Schemes. Springer-Verlag, New York, 1979. 